\title{
WARPED WAVELET BASES: UNITARY EQUIVALENCE AND SIGNAL PROCESSING
}

\author{
Richard G. Baraniuk ${ }^{\diamond}$ and Douglas L. Jones $\square$ * \\ • Laboratoire de Physique (URA 1325 CNRS) \\ Ecole Normale Supérieure de Lyon \\ 46 allée d'Italie, 69364 Lyon Cedex 07, France \\ D Coordinated Science Laboratory \\ University of Illinois, Urbana, IL 61801 , USA
}

\begin{abstract}
The notions of time, frequency, and scale are generalized using concepts from unitary operator theory and applied to timefrequency analysis, in particular the wavelet and short-time Fourier transform orthonormal bases and Cohen's class of bilinear time-frequency distributions. The result is an infinite number of new signal analysis and processing tools that are implemented simply by prewarping the signal by a unitary transformation, performing standard processing techniques on the warped signal, and then (in some cases) unwarping the resulting output. These unitarily equivalent, warped signal representations are useful for representing signals that are well modeled by neither the constant-bandwidth analysis of time-frequency techniques nor the proportional-bandwidth analysis of time-scale techniques.
\end{abstract}

\section{INTRODUCTION}

The concepts of time and frequency are the cornerstones of signal analysis and processing, for they are the basis for fundamental tools such as the Fourier transform and the linear time-invariant (LTI) system. Joint representations of both time and frequency have also been developed to indicate how the frequency content of a signal changes over time. Examples of time-frequency representations include the linear short-time Fourier transform (STFT), which can be regarded as a constant-bandwidth filterbank of LTI systems, and its generalization, Cohen's class of bilinear time-frequency distributions [1]. While these joint time-frequency representations are necessarily redundant, discretization of the STFT on a lattice of points in time-frequency can result in orthonormal basis systems whose elements are concentrated in both time and frequency [2].

While it is unlikely that time and frequency will be supplanted as the two key notions for signal processing, other concepts have also been investigated and found useful. Most notable at present are the scale-based analysis of the Mellin transform and the time-scale analysis of the wavelet transform (WT), which can be interpreted as a proportionalbandwidth (constant-Q) LTI filterbank. The WT in particular has garnered tremendous interest, mainly because it can be discretized to form time-frequency-concentrated orthonormal bases with very attractive properties [3]. The theory of bilinear time-frequency distributions has been also been generalized from time-frequency to time-scale [4], [5].

Both the time-frequency and the time-scale tools have proved useful for analyzing certain classes of signals. For

-This work was supported by an NSERC-NATO postdoctoral fellowship, the Joint Services Electronics Program, Grant No. N00014-90-J-1270, and the National Science Foundation, Grant No. MIP 90-12747. R. Baraniuk is on leave from Rice University, P.O. Box 1892, Houston, Texas 77251-1892, USA. signals with a constant-bandwidth structure, the STFT and other time-frequency tools are best suited, while for signals with a proportional-bandwidth structure, the WT and timescale tools are best suited. However, there exist large classes of signals (frequency modulated and dispersed signals are two examples) for which neither a constant-bandwidth nor a proportional-bandwidth analysis is appropriate. The goal of this paper is to derive new classes of signal representations and analysis systems that generalize the concepts of time, frequency, and scale. These systems are well suited to analyze some of the types of signals for which current techniques are deficient.

\section{UNITARY OPERATORS}

Before we can generalize the concepts of time, frequency, and scale, we must define what we mean by time, frequency, and scale. The approach we will utilize in this paper is to associate these concepts with operators ${ }^{1}$ on the Hilbert space of square-integrable functions $L^{2}$. This space has inner product $\langle g, h\rangle=\int g(\tau) h^{*}(\tau) d \tau$ for $g, h \in L^{2}$ and norm $\|h\|^{2}=\langle h, h\rangle$. An operator $\mathcal{A}$ on $L^{2}$ is simply a mapping $\mathcal{A}: L^{2} \rightarrow L^{2}$. For signals in $L^{2}$, we define the time, frequency, and scale operators simply as a time shift, $\left(\mathcal{T}_{t} g\right)(\tau)=g(\tau-t)$, frequency shift, $\left(\mathcal{F}_{f} g\right)(\tau)=e^{j 2 \pi f \tau} g(\tau)$, and dilation, $\left(\mathcal{D}_{d} g\right)(\tau)=|d|^{-1 / 2} g(\tau / d)$, respectively

We will utilize unitary operators extensively in this paper. A unitary operator $\mathcal{U}$ is a linear, surjective transformation that preserves distances; that is, $\|\mathcal{U} g\|=\|g\|$. It is easily verified that the time, frequency, and scaling operators defined above are unitary on $L^{2}$. Unitary operators have many important properties; we will introduce them (without proof - see [6] for more information) as they are required in the development.

The first important property is that a unitary operator preserves inner products; that is, $\left\langle U_{g}, U h\right\rangle=\langle g, h\rangle$. This in turn results in another useful property: a unitary operator maps orthonormal bases to orthonormal bases. ${ }^{2}$ That is, if the set of vectors $\left\{b_{k}\right\}$ is an orthonormal basis for $L^{2}$, then the set $\left\{\mathcal{U} \mathrm{b}_{k}\right\}$ is also an orthonormal basis for $L^{2}$

The most general formula for representing the unitary operators on $L^{2}$ uses the following linear superposition:

$$
(\mathcal{U} s)(\tau)=\int K_{U}(\tau, v) s(v) d v,
$$

with the requirement that the "rows" and "columns" of the integration kernel $K_{\mathcal{U}}(\tau, v)$ must both form com-

${ }^{1}$ Cohen has also associated these concepts with operators [4], but with a slightly different goal in mind.

${ }^{2} \mathrm{An}$ orthonormal basis in a Hilbert space is a complete, orthonormal set of vectors. 
plete orthonormal sets for $L^{2}$; that is, we must have $\int K_{\mathcal{U}}\left(\tau_{1}, v\right) K_{\mathcal{U}}^{*}\left(\tau_{2}, v\right) d v=\delta\left(\tau_{2}-\tau_{1}\right)$ and $\int K_{\mathcal{U}}\left(\tau, v_{1}\right)$ $K_{\mathcal{U}}^{*}\left(\tau, v_{2}\right) d \tau=\delta\left(v_{2}-v_{1}\right)$, where $\delta$ is the Dirac delta function. For example, the kernel $K_{U}(\tau, v)=e^{j 2 \pi v \tau}$ yields the Fourier transform operator $F$, whose unitarity results from Parseval's formula. A large and interesting subclass of unitary transformations on $L^{2}$ is the subclass of axis warpings that can be written as

$$
(\mathcal{U} s)(\tau)=\left|w^{\prime}(\tau)\right|^{1 / 2} s[w(\tau)],
$$

where $w$ is a smooth, monotonic function. Examples of simple yet useful warping functions include $w(\tau)=\log \tau, w(\tau)=e^{\tau}$, and $w(\tau)=\operatorname{sign}(\tau)|\tau|^{k}, k \neq 0$.

Because a unitary operator maps $L^{2}$ back onto itself in a way that preserves exactly its structure - it does not change the distances or angles between vectors - unitary transformations can be interpreted as simply "relabeling operators" that take every function $s \in L^{2}$ and give it a new name $\mathcal{U} s$ This relabeling is equivalent to changing the frame of reference or changing bases. (For example, recall that the unitary operators on ordinary Euclidean space $\mathbb{R}^{n}$ are simply rotations around some axis.) Applying the same reasoning to the linear transformations on $L^{2}$ leads to the following definition of operators that are equivalent modulo a change of basis.

Definition 1 Two operators $\mathcal{A}$ and $\mathcal{B}$ are unitarily equivalent if we can write $B=U \mathcal{A} \mathcal{V}$, where $\mathcal{U}$ and $\mathcal{V}$ are unitary transformations.

By analogy to (2), if $\mathcal{A}$ and $\mathcal{B}$ are unitarily equivalent, then we will say that $\mathcal{B}$ is a warped version of $\mathcal{A}$. Note that by considering the operator $\mathcal{U A \mathcal { V }}$ for all possible unitary transformations $\mathcal{U}$ and $\mathcal{V}$, we can construct equivalence classes of operators that are unitarily equivalent. In this paper, we will set $\mathcal{V}$ equal to $\mathcal{U}^{-1}$ or to the identity operator (both of which are unitary) for constructing these equivalence classes. We will find this concept very useful for generalizing the concepts of time, frequency, and scale in linear systems, mapping them to new concepts that can better match a class of signals to be analyzed.

\section{WARPED WAVELET BASES}

As a first example of the utility of unitary equivalence, we now generalize the time-frequency-concentrated orthonormal bases that arise from the discretization of the STFT and WT. ${ }^{3}$ Given a doubly indexed orthonormal basis $\left\{\mathbf{b}_{m, n}\right\}$ for $L^{2}$, we can decompose any signal $s \in L^{2}$ as follows:

$$
s=\sum_{m, n \in Z}\left\langle s, \mathbf{b}_{m, n}\right\rangle \mathbf{b}_{m, n} \text {. }
$$

The elements of an STFT basis are obtained by translating and modulating a nonarbitrary but fixed window function $g_{\text {stft }}[2]$

$$
\begin{aligned}
\mathbf{b}_{m, n}^{\text {stft }}(\tau) & =\left(\mathcal{F}_{n f_{0}} \mathcal{T}_{m t_{0}} g_{\mathrm{stft}}\right)(\tau) \\
& =g_{\mathrm{stft}}\left(\tau-m t_{0}\right) e^{j 2 \pi n f_{0} \tau}
\end{aligned}
$$

The basis elements can be interpreted as "tiling" the timefrequency plane in a constant-bandwidth fashion; the tiling for an idealized STFT basis is depicted in Fig. 1. The elements of a WT basis are obtained by translating and scaling a nonarbitrary but fixed wavelet function $g_{\mathrm{w} t}[3]$

$$
\mathbf{b}_{m, n}^{\mathrm{wt}}(\tau)=\left(\mathcal{T}_{m t_{0} d_{0}^{n}} \mathcal{D}_{d_{0}^{n}} g_{\mathrm{wt}}\right)(\tau)
$$

${ }^{3}$ Note that these techniques can be applied to bases other than those arising from the STFT and WT without modification, as well as to frames in $L^{2}$ [3].

$$
=d_{0}^{-n / 2} g_{\mathrm{wt}}\left(d_{0}^{-n} \tau-m t_{0}\right) \text {. }
$$

These basis elements tile the time-frequency plane in a proportional-bandwidth fashion; the tiling for an idealized WT basis is pictured in Fig. 2

With the STFT and WT, we have two disparate tilings of the time-frequency plane that are each well suited for representing certain classes of signals. However, as discussed above, what if the signals we wish to decompose are not well modeled by a constant-bandwidth or a proportionalbandwidth analysis? For example, the energy of a frequency modulated (FM) signal will be spread over many basis coefficients in both types of expansions, since it traces a path in the time-frequency plane which is not well modeled by either of the basis tilings shown in Figs. 1 and 2 .

While the STFT or WT bases may not be the most appropriate for representing certain classes of signals, there may exist unitarily equivalent bases that are appropriate. Furthermore, these bases are simple to generate. Consider the effect of operating on each the elements of the STFT and WT bases with a unitary transformation $U$. Since $\mathcal{U}$ is unitary, both $\left\{\mathcal{U} b_{m, n}^{\text {stft }}\right\}$ and $\left\{\mathcal{U} b_{m, n}^{\mathrm{wt}}\right\}$ are also valid orthonormal bases for $L^{2}$; we will refer to them as warped bases. ${ }^{4}$ However, while the index parameters $m$ and $n$ represent time and frequency in the STFT bases and time and scale in the WT bases, they are mapped to new concepts in the warped bases. To see this, we can write for the STFT basis

$$
\begin{aligned}
\mathcal{U} \mathrm{b}_{m, n}^{\mathrm{stft}} & =\mathcal{U}\left(\mathcal{F}_{n f_{0}} \mathcal{T}_{m t_{0}} g_{s \mathrm{ft}}\right) \\
& =\left(\mathcal{U} \mathcal{F}_{n f_{0}} \mathcal{U}^{-1}\right)\left(\mathcal{U} \mathcal{T}_{m t_{0}} \mathcal{U}^{-1}\right)\left(\mathcal{U} g_{\mathrm{stft}}\right)
\end{aligned}
$$

and for the WT basis

$$
\begin{aligned}
\mathcal{U} \mathrm{b}_{m, n}^{\mathrm{wt}} & =\mathcal{U}\left(\mathcal{T}_{m t_{0} d_{0}^{n}} \mathcal{D}_{d_{0}^{n}} g_{\mathrm{stft}}\right) \\
& =\left(\mathcal{U} \mathcal{T}_{m t_{0} d_{0}^{n}} \mathcal{U}^{-1}\right)\left(\mathcal{U} \mathcal{D}_{d_{0}^{n}} \mathcal{U}^{-1}\right)\left(\mathcal{U} g_{\mathrm{wt}}\right)
\end{aligned}
$$

Thus, the effect of $\mathcal{U}$ is to warp the original time, frequency, and scale operators to three new unitarily equivalent operators, and to map the original window $g_{\text {stft }}$ and wavelet $g_{w t}$ to two new functions. The key to this procedure is clearly to choose $\mathcal{U}$ so that the warped basis better matches the characteristics of the signals at hand.

As an example, consider the unitary transformation $U=\mathbf{F}^{-1} \Lambda_{k} \mathbf{F}$, where $\Lambda_{k}$ is an axis warping defined as in (2) with $w(\tau)=\operatorname{sign}(\tau)|\tau|^{k}, k \neq$ 0 [7]. Operating on an arbitrary WT basis (5) with $\mathcal{U}$ results in a new basis with elements $\mathcal{U} \mathbf{b}_{m, n}^{w t}(\tau)=$ $\left(-j k m t_{0}\right)^{-\frac{1}{2}}\left[d_{0}^{-n / k} \tilde{g}\left(d_{0}^{-n / k} \tau\right) \star e^{-j\left(a_{0}^{-n / 2 k} \tau\right)^{k} / 4 m t_{0}}\right]$, where $\tilde{g}=\mathcal{U}_{g_{\mathrm{w}}}$ and $\star$ denotes convolution. Time and scale in the original basis are mapped to chirp rate $m$ and scale $n$, re spectively. The idealized tiling in the time-frequency plane for this basis, shown in Fig. 3 for the case $k=2$, is quite different from the STFT and WT tilings of Figs. 1 and 2.

Note from (6) and (7) that since $\mathcal{U}$ works on both the time and frequency or time and scale operators simultaneously, there exists a tradeoff in its choice: any changes that we make to one operator are also imparted in the other. Thus, special choices of unitary maps can prove useful. For example, the $|\tau|^{k}$ warp employed above is interesting for WT bases, because it essentially commutes with the scale operator. In the next section, we will study in more detail the physical signif-

${ }^{4}$ Note that the problem as posed for time-frequency and timescale is equivalent to characterizing all unitarily equivalent representations of the Weyl-Heisenberg and affine groups. 
icance of the warped time, frequency, and scale operators.

One possible problem with the warped bases of (6) and (7) is that the coefficients of the warped expansions may be cumbersome to compute. However, the unitarity of $U$ allows us to compute the warped coefficients by first unitarily prewarping the signal and then computing the usual coefficients. That is, the coefficients of a warped STFT basis expansion are given by

$$
\begin{aligned}
\left\langle s, \mathbf{b}_{m, n}^{\text {warped }}\right\rangle & =\left\langle\mathcal{U}^{-1} s, \mathcal{U}^{-1} \mathbf{b}_{m, n}^{\text {warped }}\right\rangle \\
& =\left\langle\mathcal{U}^{-1} s, \mathbf{b}_{m, n}^{\text {stf }}\right\rangle,
\end{aligned}
$$

with a similar calculation for the WT bases. While indicating that warped basis expansions can be implemented just as efficiently (modulo the unitary preprocessing) as the original basis expansions, this computation also emphasizes that warping a basis set to match a signal is equivalent to prewarping the signal to match an existing basis.

\section{WARPED GEOMETRIES FOR SIGNAL} PROCESSING

The scope of the previous development is not limited to warping only STFT and WT bases. In this section, we replace the basis coefficient calculation (8) with an arbitrary LTI system $^{5}$ and investigate the properties of unitarily equivalent LTI systems. An LTI system $\mathcal{P}$ is a linear operator on $L^{2}$ that transforms signals through convolution with a onedimensional function. Given an LTI system $\mathcal{P}$, we will consider the equivalence class of systems given by $\mathcal{P} U^{-1}$, where $U$ is an arbitary unitary operator on $L^{2}$. Note that this is equivalent to preprocessing the input signal by $\mathcal{U}^{-1}$ before feeding it into the system $\mathcal{P}$ - in effect changing bases or frames of reference. ${ }^{6}$ By picking the transformation $\mathcal{U}$ intelligently, it will be possible to analyze and process certain classes of signals more efficiently than with traditional LTI techniques alone.

\subsection{Eigenanalysis of Linear Systems}

Eigenanalysis is a powerful tool for studying the characteristics of linear operators. Given a linear operator $\mathcal{A}$ on $L^{2}$ solution of the eigenequation

$$
\left(\mathcal{A} u_{a}\right)(\tau)=\lambda_{a} u_{a}(\tau)
$$

yields the eigenfunctions $\left\{u_{a}(\tau)\right\}$ and the eigenvalues $\left\{\lambda_{a}\right\}$ of $\mathcal{A}$, both of which are indexed by the parameter $a$. If $\mathcal{A}$ is unitary, then the eigenfunctions form a complete orthonormal set for $L^{2}$, and we can can define another unitary transform, which we will refer to as the $\mathcal{A}$-Fourier transform $\boldsymbol{F}_{\mathcal{A}}$. The forward transform is given by

$$
S(a)=\left(\mathrm{F}_{A} s\right)(a)=\left\langle s(\tau), u_{a}(\tau)\right\rangle,
$$

while the inverse transform is given by

$$
s(\tau)=\left(\mathbf{F}_{A}^{-1} S\right)(\tau)=\left\langle S(a), u_{a}^{*}(\tau)\right\rangle
$$

(in an abuse of notation, we retain the index of the functions inside the inner product symbol to indicate the variable of integration $-\tau$ in (10) and $a$ in (11))

${ }^{5}$ Identical techniques can be applied to linear time-varying systems; however, space limitations preclude their inclusion in this paper.

${ }^{6}$ The application of unitary equivalence to systems is not a new concept, and has been used to great advantage in a number of applications, including transform coding, transform domain adaptive filtering, "beamspace" sensor array processing, and dynamic time-warping of speech signals, However, this paper represents the first application of this technique to general time-frequency and time-scale systems.

\subsection{Eigenanalysis of LTI Systems}

Eigenanalysis provides a natural justification that the time and frequency operators defined in Section 2 are the fundamental operators for LTI systems. The time operator is obviously fundamental, since, by definition, an LTI system $\mathcal{P}$ is invariant ${ }^{\gamma}$ to time shifts; that is $\left(\mathcal{P} \mathcal{T}_{t} s\right)(\tau)=\left(\mathcal{T}_{t} \mathcal{P}_{s}\right)(\tau)=$ $\left(\mathcal{P}_{s}\right)(\tau-t)$. Furthermore, since $\mathcal{P}$ and $\mathcal{T}_{t}$ commute, they share a common set of eigenfunctions, which are easily shown to be the complex exponentials $u_{f}(\tau)=e^{j 2 \pi f r}$ - precisely the weighting functions in the frequency-shift operator $\mathcal{F}_{f}$.

Thus, the concept of time can be defined abstractly as the parameter of the time-shift operator $\tau_{t}$, and the concept of frequency can be defined abstractly as the index parameter of the eigenfunctions of $\mathcal{T}_{t}$ (and therefore also as the parameter of the $\mathcal{T}_{t}$-Fourier transform, which in this case is the usual Fourier transform.)

\subsection{Eigenanalysis of Warped LTI Systems}

What happens to the fundamental concepts of time and frequency after we place a unitary prewarping at the input of an LTI system? This problem can be solved using the same procedure as above. A simple calculation shows that since $\mathcal{P}$ is invariant to $\mathcal{T}_{t}$, the prewarped system $\mathcal{P U} \mathcal{U}^{-1}$ is invariant to the operator $U \mathcal{T}_{t}, U^{-1}$; that is, $\left[\left(\mathcal{P} U^{-1}\right)\left(U \mathcal{T}_{t^{\prime}} U^{-1}\right) s\right](\tau)=\left(\mathcal{P} \mathcal{U}^{-1} s\right)\left(\tau-t^{\prime}\right)$. Therefore the time operator and the concept of time are warped to the operator $\mathcal{U} \mathcal{T}_{t^{\prime}} \mathcal{U}^{-1}$ and its parameter $t^{\prime}$. Moreover, the eigenfunctions of $u \tau_{t}, \mathcal{U}^{-1}$, which correspond to the warped frequency operator and warped concept of frequency, are given by $\left(U u_{f^{\prime}}\right)(\tau)$, where $u_{f^{\prime}}(\tau)=e^{j 2 \pi f^{\prime} \tau}$. Note that the warped time and warped frequency domains are related by the $\mathcal{U} \mathcal{T}_{t} \mathcal{U}^{-1}$-Fourier transform, which for $\mathcal{U}$ from the axis warp subclass is essentially the FAM transform [8]. (See also [9] for another approach to generalized Fourier transforms.)

Thus, given an LTI system $\mathcal{P}$, for each choice of unitary operator $\mathcal{U}$, we generate a new linear system $\mathcal{P} \mathcal{U}^{-1}$ with generalized notions of time and frequency. Furthermore, by the "relabeling property" of $\mathcal{U}$, all familiar LTI properties - support, bandwidth, and group delay are examples -- are remapped to new properties that change with $\mathcal{U}$. The key issue is therefore to choose $\mathcal{U}$ so that these quantities correspond to something meaningful for the signal class of interest.

As a first example, consider the axis warping unitary transformation $\mathcal{U}_{1}$ defined as in (2) with $w(\tau)=\log \tau$. For singlesided signals $(s(\tau)=0, \tau<0)$, this transformation maps the time operator $\mathcal{T}_{t}$ to the scale operator $\mathcal{D}_{e t}$, and thus transforms an LTI system to a linear scale-invariant system [4]. The eigenfunctions of such a system are the "hyperbolic chirp" functions $h_{c}(\tau)=\tau^{-1 / 2} e^{j 2 \pi c \log \tau}$, whose parameter $c$ corresponds to warped frequency. The $\mathcal{U}_{1} \tau_{t} \mathcal{U}_{1}^{-1}$-Fourier transform in this case is the Mellin transform.

As a second example, consider the axis warping unitary transformation $U_{2}$ defined as in (2) with $w(\tau)=\tau^{2}$. For onesided signals, this transformation maps the time operator $\mathcal{T}_{t}$ to the operator $\left(\widetilde{T}_{t^{\prime}} g\right)(\tau)=2^{-1 / 2}\left(\tau^{2}-t^{\prime}\right)^{-1 / 4} g\left(\sqrt{\tau^{2}-t^{\prime}}\right)$, a kind of time-shift/warping combination, to which the system $\mathcal{P U} \mathcal{L}^{-1}$ is invariant. The eigenfunctions of $\widetilde{T}_{t^{\prime}}$, computed as $\left(\mathcal{U}_{2} u_{f^{\prime}}\right)(\tau)$, where $u_{f^{\prime}}(\tau)=e^{j 2 \pi f^{\prime} \tau}$, are the linear-FM chirp functions $(2 \tau)^{1 / 2} e^{j 2 \pi f^{\prime} \tau^{2}}$. The $\mathcal{U}_{2} \mathcal{T}_{t} \mathcal{U}_{2}^{-1}$-Fourier transform in this case represents signals in terms of these chirp functions.

${ }^{7}$ Covariant is actually a more accurate term for this property. 


\subsection{Warped Time-Frequency Systems}

A very useful application of unitary prewarping is the construction of unitary equivalence classes of time-frequency distributions (TFDs). We will outline the procedure for Cohen's class of bilinear TFDs [1], which can be considered as a generalization of single-input, multiple-output LTI systems. For full details, see [10].

Let $(\mathcal{C} s)(\tau, \nu)$ denote a bilinear mapping from signals $s \in$ $L^{2}$ to the time-frequency plane, with $\tau$ and $\nu$ representing time and frequency, respectively. Cohen's class is defined as the set of all such functionals that are invariant to both the time and frequency shift operators [1]; that is, for each TFD in Cohen's class we have $\left(\mathcal{C F}_{f} \mathcal{T}_{t} s\right)(\tau, \nu)=(\mathcal{C} s)(\tau-t, \nu-f)$. In addition to being invariant to the time and frequency operators, these TFDs are also fixed for the eigenfunctions of these operators. That is, for the time operator, the eigenfunctions are sinusoids $u_{f}(\tau)=e^{j 2 \pi f \tau}$, and we have $\left(\mathcal{C T}_{t} u_{f}\right)(\tau, \nu)=$ $\left(\mathcal{C} u_{f}\right)(\tau, \nu)$. For the frequency operator, the eigenfunctions are easily shown to be impulses $u_{\mathrm{t}}(\tau)=\delta(\tau-t)$, and we have $\left(\mathcal{C} \mathcal{F}_{f} u_{t}\right)(\tau, \nu)=\left(\mathcal{C} u_{t}\right)(\tau, \nu)$. Some TFDs from Cohen's class have the marginal properties $\int(\mathcal{C} s)(\tau, \nu) d \nu=|s(\tau)|^{2}$ and $\int\left(\mathcal{C}_{s}\right)(\tau, \nu) d \nu=|(\mathbf{F} s)(\nu)|^{2}$, and all can be computed via a 2-d Fourier transform of a symmetrized form of the ambiguity function (AF) of the signal $A(\alpha, \beta)=\left(s, \mathcal{F}_{\alpha} \mathcal{T}_{\beta} s\right\rangle$

The application of a unitary prewarping to Cohen's class remaps all of these properties, but in predictable and potentially useful ways. We will call the unitarily equivalence class of bilinear distributions generated by $\mathcal{C U}^{-1}$ the $\mathcal{U}$-Cohen's class. It is easily shown that distributions in a $\mathcal{U}$-Cohen's class have the following properties [10]: 1) they are invariant to the operators $\mathcal{U} \mathcal{T}_{t} \mathcal{U}^{-1}$ and $\mathcal{U} \mathcal{F}_{f} \mathcal{U}^{-1}$, and therefore the $U$-Cohen's class consists of distributions not of time and frequency, but of warped time and warped frequency; 2) the signals for which they are fixed are precisely the eigenfunctions $\mathcal{U} u_{f}$ and $\mathcal{U} u_{t}$ of the warped time and frequency operators; 3$)$ their marginals are remapped to $\int\left(\mathcal{C U}^{-1} s\right)(\tau, \nu) d \nu=\left|\mathcal{U}^{-1} s(\tau)\right|^{2}$ and $\left.\int\left(\mathcal{C U}^{-1} s\right)(\tau, \nu) d \nu=\left|\left(\mathrm{F}_{U} s\right)(\nu)\right|^{2} ; 4\right)$ they can be computed as the 2-d Fourier transform of a symmetrized form of the $\mathcal{U}-\mathrm{AF}$ of the signal $A_{\mathcal{U}}(\alpha, \beta)=\left(s,\left(\mathcal{U} \mathcal{F}_{\alpha} \mathcal{U}^{-1}\right)\left(\mathcal{U} \mathcal{T}_{\beta} \mathcal{U}^{-1}\right) s\right)$. As with the warped STFT bases of (6), $U$ warps both time and frequency in the same fashion, so care has to be taken to balance the effect of $\mathcal{U}$ on both variables.

Continuing the examples of the previous section, we see that the $U_{2}$-Cohen's class corresponds to joint bilinear distributions of "time" and linear chirp rate. On the other hand, the unitary operator $F U_{1} \mathbf{F}^{-1}$ maps the frequency operator to the scale operator and the time operator to a "hyperbolic time shift." Thus, the $F \mathcal{U}_{1} \mathbf{F}^{-1}$-Cohen's class corresponds (within a simple variable transformation) to the proportionalbandwidth analysis hyperbolic class introduced recently in [5] and independently in [4].

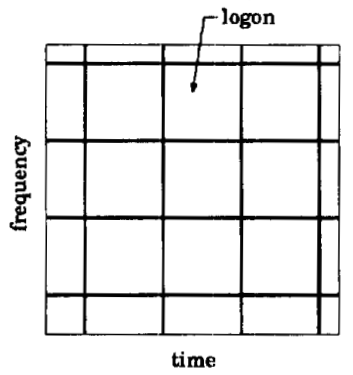

Fig. 1: STFT basis tiling.

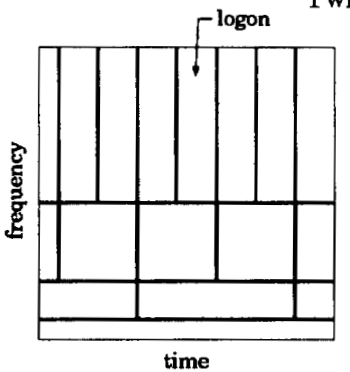

Fig. 2: WT basis tiling.

\section{CONCLUSIONS}

Using unitary operators, we have developed an infinite number of new signal analysis and processing tools that go beyond the confines of a strict time-frequency or time-scale analysis. These new tools are very easily implemented simply by prewarping the signal by a unitary transformation, performing standard processing techniques on the warped signal, and then (in some cases) unwarping the resulting output. Note that while we emphasized the time-frequency analysis of Cohen's class in the last section, we can also warp time-scale (as we did for the WT orthonormal bases) and other systems. The only limitation to this procedure is that the effect of the unitary operator is distributed equally on all analysis variables of the original system (see (6), for example). Thus, while a warping may result in desirable properties for the warped system (scale invariance, for example), it may also result in a loss of some of the desirable properties of the original system (time-shift invariance, for example). In spite of this possible limitation, there do exist warped systems that are useful; the hyperbolic class mentioned above is one example. In the future, we can imagine the choice of a warping operator being made automatically to best fit a certain style of analysis to a given set of data.

\section{REFERENCES}

1. L. Cohen, "Time-Frequency Distributions - A Review," Proc. IEEE, Vol. 77, No. 7, 1989.

2. H. Jensen et al., "Double Series Representation of Bounded Signals," IEEE Trans. Information Theory, IT34, No. 4. pp. 613-624, 1988.

3. C. Heil and D. Walnut "Continuous and Discrete Wavelet Transforms," SIAM Review, Vol. 31, No. 4, pp. 628-666.

4. L. Cohen, "A General Approach to Obtaining Joint Representations in Signal Analysis and an Application to Scale", Proc. SPIE 1566, San Diego, July 1991.

5. A. Papandreaou, et al., "A Unified Framework for the Bertrand Distribution and the Altes Distribution: The New Hyperbolic Class of Quadratic Time-Frequency Distributions," IEEE-SP Symp. Time-Frequency and TimeScale, Oct. 1992

6. F. Riesz and B. Sg.-Nagy, Functional Analysis, Frederick Ungar Pub. Co., New York, 1955.

7. R. Baraniuk and D. Jones, "New Signal-Space Orthonormal Bases Via the Metaplectic Transform," IEEE-SP Symp. Time-Frequency and Time-Scale, Oct. 1992.

8. U. Laine, "FAMlet, To Be or Not To Be a Wavelet?" IEEESP Symp. Time-Frequency and Time-Scale, Oct. 1992.

9. S. Puechmorel and B. Lacaze, "Optimal Transforms Related to Paths in the Time-Frequency Plane," IEEE-SP Symp. Time-Frequency and Time-Scale, Oct. 1992.

10. R. Baraniuk and D. Jones, "Unitary Equivalence: A New Twist on Signal Processing," preprint.

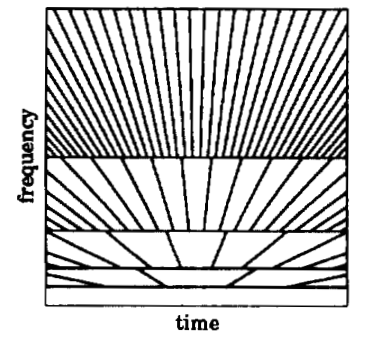

Fig. 3: Warped WT basis tiling.

III-323 